Volume 8. No. 9, September 2020

International Journal of Emerging Trends in Engineering Research

Available Online at http://www.warse.org/IJETER/static/pdf/file/ijeter219892020.pdf

https://doi.org/10.30534/ijeter/2020/219892020

\title{
Driver Fatigue and Alcohol Detection System for Prevention of Accidents
}

\author{
S. Swathi ${ }^{1}$, Mr. T. Ramakrishnaiah ${ }^{2}$ \\ ${ }^{1}$ Student, Department of Electronics and Communication Engineering, Vardhaman College of Engineering, \\ Hyderabad, India, sherla.swathi@gmail.com \\ ${ }^{2}$ Assistant Professor, Department of Electronics and Communication Engineering, Vardhaman College of \\ Engineering, Hyderabad, India, t.ramakrishnaiah@ vardhaman.org
}

\begin{abstract}
Automobile disasters are majorly caused by the fatigue of the driver which is due to continual restless driving for a longer period or driving the vehicle after consumption of alcohol. This type of accident leads to many fatalities and severe injuries. Therefore, a countermeasure device has to be used to prevent such types of road accidents, thus the demand for creating the fatigue and alcohol detection system is high. This paper provides an eye monitoring system that alerts fatigue detected driver using buzzer and stops the vehicle during the state of drowsiness. The information of the driver state will also be sent along with the location. If alcohol is detected it locks the vehicle engine to stop the automobile and the location of the alcohol detected vehicle is sent to a respective person. The person might be traffic authorities or to a family member, so that they can take the necessary measure to reduce the risk of accidents.
\end{abstract}

Key words: Buzzer, fatigue, driver monitoring, alcohol detection.

\section{INTRODUCTION}

In India, most of the deaths are caused due to road accidents. The primary reason for these accidents is Driver's fatigue, here fatigue is defined as extreme tiredness and the reasons for fatigue may be working or driving for long period. The result of fatigue is mental or physical effort or illness or drowsiness [1]. According to National Informatics Center [2], the accident rate is been increased in the last five years and the major reasons for the cause of accidents are 1.Human error 2.Road environment and 3.Vehicular condition.

Human error includes the violation of the traffic rules, drunken driving, loss of control of the vehicle at high-speed \& use of mobile phones while driving. The reports also include that these accidents are caused by the age group of 18-35.
Road environment includes the accidents caused by Weather conditions, road features including curved, straight, steep road, and junction type.

Vehicular condition include the age of the vehicle, load it carries because the heavy loaded vehicle may cause the tyres to burst and condition of brakes and parts.

To control the death rate caused due to road accidents our primary concern is to prevent before it occurs by monitoring driver's fatigue and detecting the alcohol in the Vehicle using an effective system. The fatigue and alcohol consumption by the driver does great harm to public security, so this system has a preventative effect that can stop accidents from occurring and concentrates on human's safety.

The Driver fatigue can be detected by monitoring his/her symptoms of drowsiness like measuring the eye blinks and Alcohol sensor for detecting the alcohol in Vehicle. When the system detects the drowsiness or alcohol a buzzer is blown and the engine will be locked thus preventing the accidents to being occurred.

Moreover, pedestrians or people in other vehicle will be much safer because the detected vehicle is been stopped right away and information regarding detection is given to the Traffic authority or respective person for necessary action to be taken.

\section{LITERATURE SURVEY}

Bappaditya Mandal, Liyuan Li et al [1] discusses the way for monitoring the driver's fatigue using image processing by detecting the head-shoulder, face, eye and calculating the PERCLOS. This system involves a camera for capturing/monitoring the driver's fatigue and detecting mentioned elements using Open CV, Python, and dlib library.

Pranjali Ingalepatil, Priyanka Barhate, et al [3] proposed system involves an alcohol sensor interfaced with Arduino for the detection of alcohol. If alcohol has detected a buzzer is blown and the ignition of the vehicle is stopped. 
S. Swathi et al., International Journal of Emerging Trends in Engineering Research, 8(9), September 2020, 6275 - 6279

Nawaz D [4] discussed about recognition of face using MAT lab. This method benefits the organization to avoid duplication of candidates in an interview process.

Teresa Soukupova and JanCech [5] discussed an algorithm for the detection of eye blink in video and calculation of the EAR which you use to detect the drowsiness of a person.

NamitaShinde, Amresh Giri et al [6] proposed a system that involves an alcohol sensor interfaced with Arduino. The sensor detects the alcohol present in the breath of the driver. When the Alcohol is detected the buzzer beeps and the engine of the vehicle is stopped.

Aaron Don, Francis Xavier et al [7] discussed about location identification in mobile devices using the GPS system. This is applicable in many tracking applications that make beneficial to society in terms of safety of a person.

\section{DESIGN AND EXECUTION}

\section{A. Hardware Design}

Raspberry pi is interfaced with an Alcohol sensor, GSM module, buzzer, motor, GPS module, and a camera. The camera is for capturing the images and live-stream the video of the driver, which is used for drowsiness detection. The alcohol detection is through alcohol sensor and the buzzer will give the alert of detection. GSM module for giving the detection message for authorized person and motor is used to lock the engine of the vehicle. The system modules are shown below.

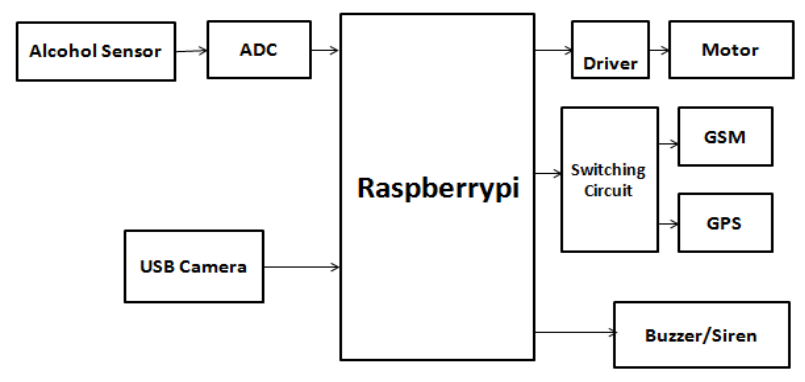

Figure 1: Hardware design of system

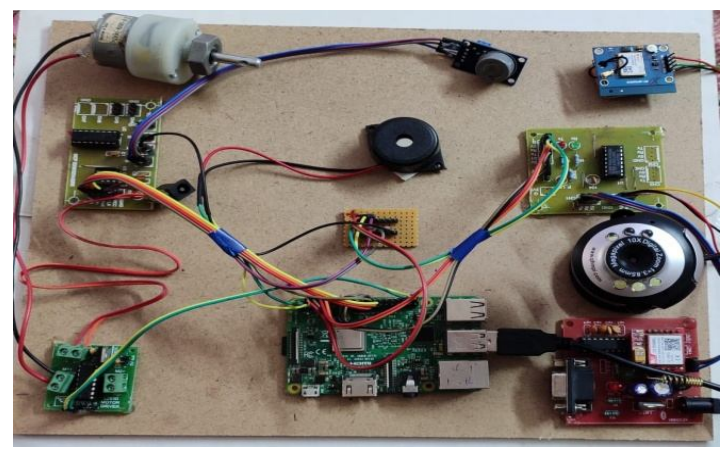

Figure 2: Setup of designed system

\section{1) Camera}

A USB camera is connected to the raspberry pi. They are plenty of cameras obtainable in retail but we choose a camera with affordable cost along with good resolution. Its role is to detect the eye blinks additionally to live-stream the driver's eye.

\section{2) Alcohol sensor}

It is just like common breath-analyzer which is suitable for detecting alcohol in breath. It has an analog and digital pin, $\mathrm{Vcc}$ and GND. The analog values from the sensor are given to the ADC module, which is converted to digital and given to raspberry pi. It will process the related action if alcohol is detected. In my project on detection of alcohol, it will lock the vehicle and the detected message is sent.

\section{3) Location Information}

The GPS module and GSM module is interfaced with the Raspberry pi for sending the information of location along with the detected message of Alcohol for Traffic management.

\section{4) Locking the Engine}

If drowsiness or Alcohol is detected by the system, then the engine will be locked. This is prototyped by interfacing a motor to Raspberry pi.

\section{B. Software Model}

Raspberry pi should be installed with OpenCV and dlib library for performing image processing operations to detect the eye blinks and for calculating the EAR (Eye Aspect Ratio).

Detecting eyes, nose, and lips \& with dlib, openCV and python:

After installing the OpenCV, python and dlib library in PC the detection can done in two ways:

a. Locating the face index in image: we use Haar cascades which are in-built in OpenCV's (for object detection)

b. Area of interest is detected in the face(like eyes): We use dlib library which is already included with facial-landmark detector

1) About dlib's facial landmark detector:

The 68(x,y) coordinates of facial-landmarks are used to map the face location. 


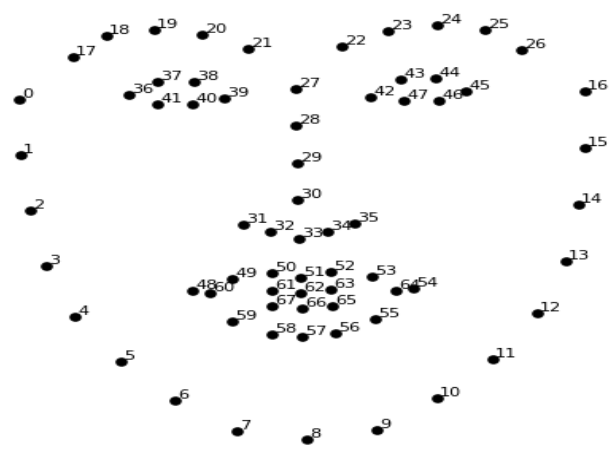

Figure 3: Visualizing facial-landmarks.

\section{2) Detection of eye blinks}

To construct the eye blink-detector, EAR should be computed. It is the ratio calculated between distances of eye in facial-landmark.

As shown in the Figure 4 each eye as 6 coordinates, starting from P1 to P6 the notation for the eye is given in clockwise manner.
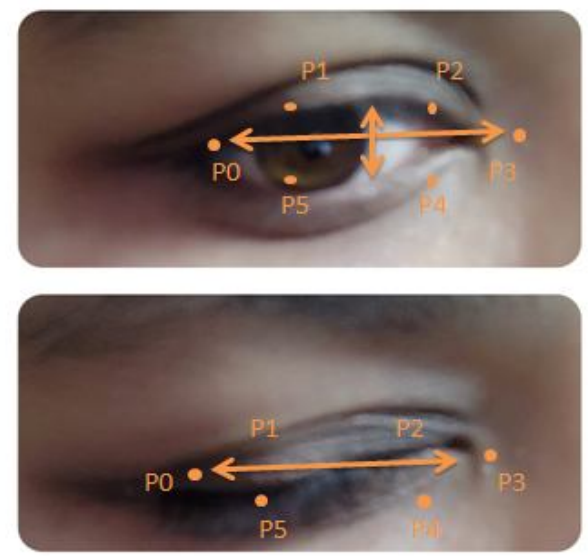

Figure 4: Visualization of Eye with 6 coordinates

When eye is wide EAR value is constant but varies and become nearly equal to zero when a blinking is taking place, thus we can determine the drowsiness by estimating the eye blinks. It can be determined using equation "(1)".

$$
\mathrm{EAR}=(\|\mathrm{P} 1-\mathrm{P} 5\|+\| \mathrm{P} 2-\mathrm{P} 4||) / 2|| \mathrm{P} 0-\mathrm{P} 3 \|
$$

\section{Flowchart}

The proposed system's flow of operation is represented in the flowchart.

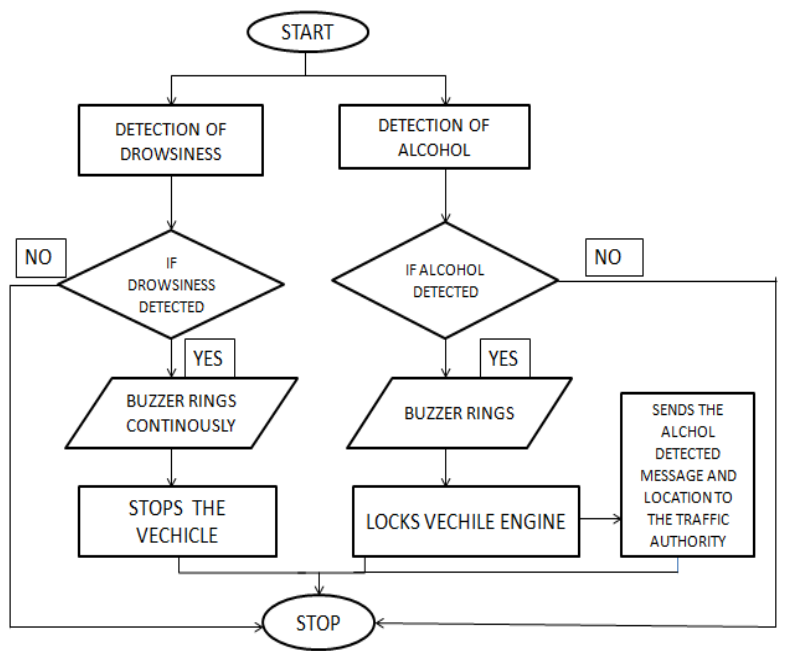

Figure 5: Workflow of Proposed system

\section{EXPERIMENTAL RESULTS}

The experimental results are shown below with diagrams.

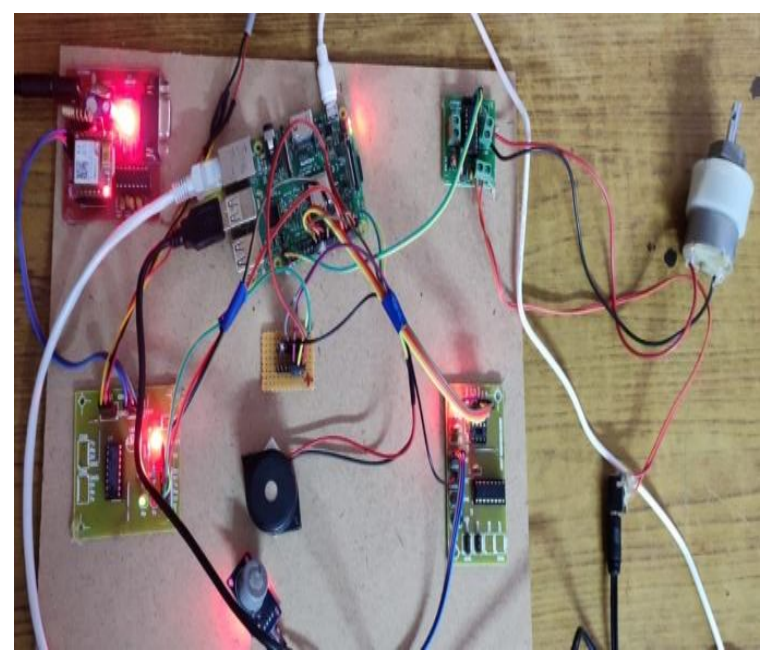

Figure 6: Setup of the proposed system

\section{A. Fatigue detection output}

After turning on the system the camera will live stream the video of driver and detect the eyes as show in the Figure 7. The blinks are counted and calculation of EAR is done using "(1)". If the value of EAR is nearly equal to zero then the fatigue is detected .Buzzer is blown to alert driver and detected message along with the location is sent which is shown in the Figure 11. 


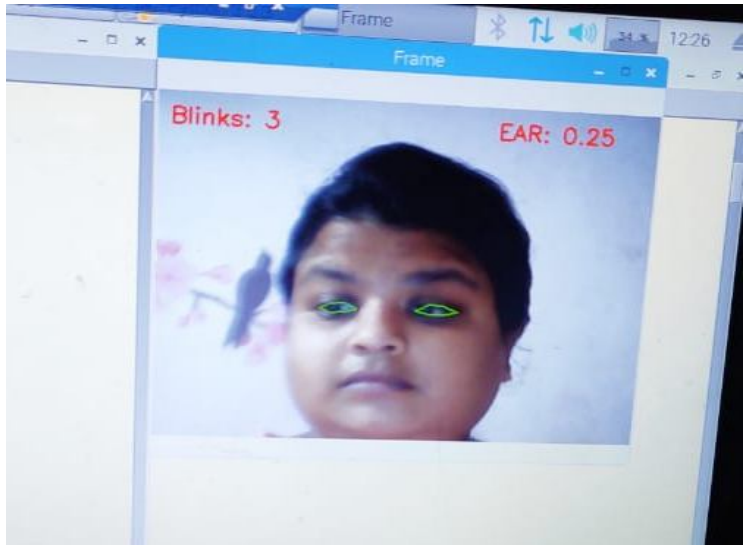

Figure 7: Image displaying the Eye's Detection with EAR value and blink count

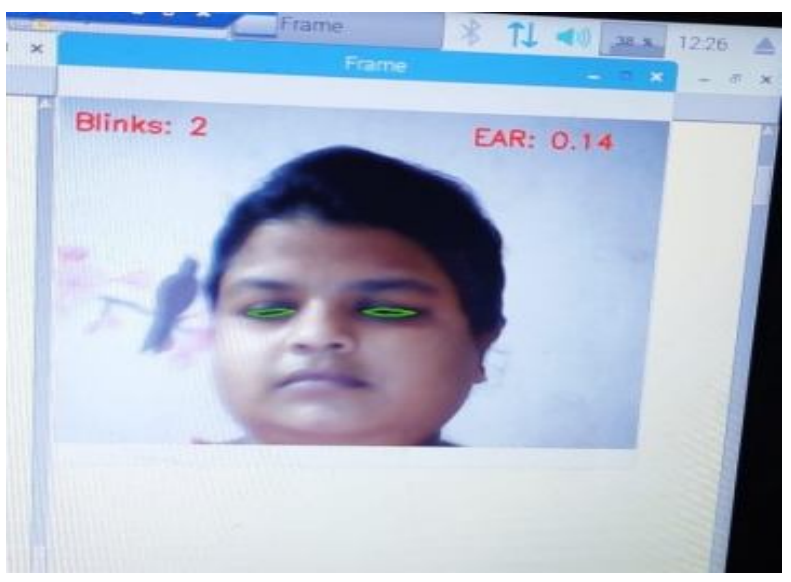

Figure 8: Image display explains the variation of EAR when eyes are closed

\section{B. Alcohol detection}

When the system is turned $\mathrm{ON}$, it monitors fatigue along with alcohol content in atmosphere. If alcohol is present in the air or breathe the reading will be more than 400 indicating alcohol detection as shown in Figure 10. The engine will be locked and detected message is sent along with location to track the vehicle Figure 11.

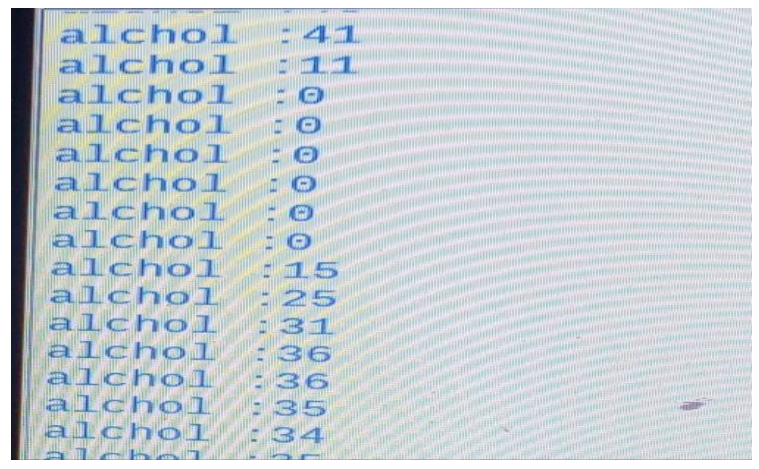

Figure 9: Image displaying readings of alcohol content

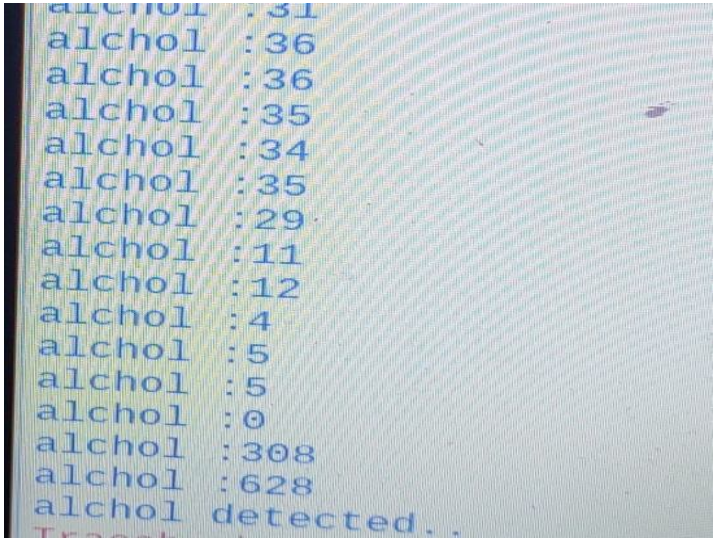

Figure 10: Image displaying variation in reading after alcohol detection

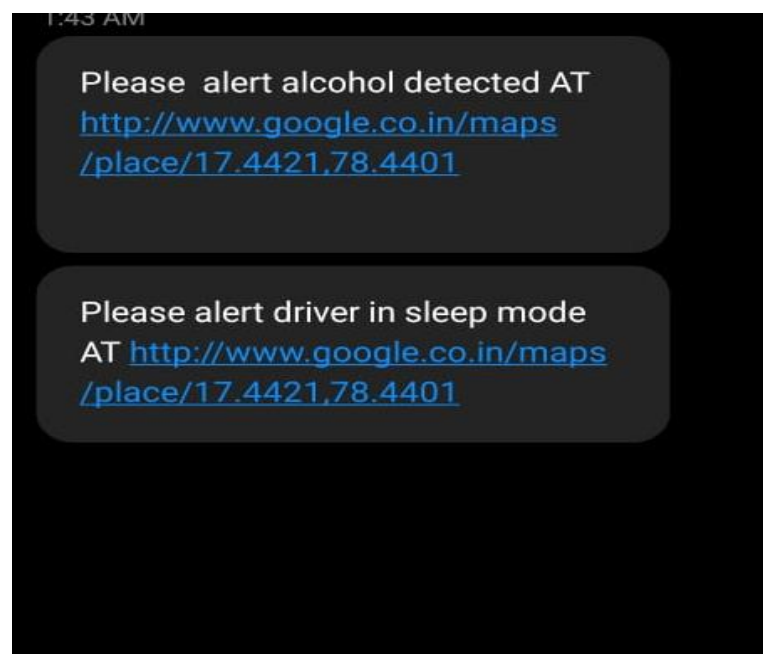

Figure 11: Message of drowsiness and alcohol detection

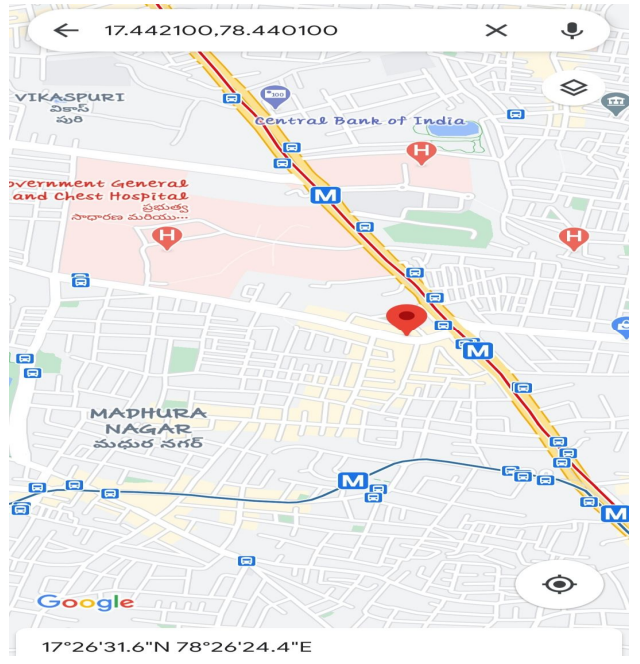

Figure 12: Location of detected vehicle 


\section{CONCLUSION}

In the paper, we have reviewed about the existing systems and using them we had implemented our system to prevent the accidents. In the proposed system, we had added more features to the existing prototype by including the GPS and GSM module for sending the detected vehicle information so, that we can track the vehicle and take the necessary action.

A camera is used to live stream the video of the driver. Using the algorithm of image processing the eye blinks and EAR is calculated and fatigue is being detected and Alcohol sensor is being used to detect alcohol in the breath.

After detecting, as the vehicle is stopped immediately this prevents the accidents to happen with the state of the driver. In this way, we can reduce the accident rate and increase public safety.

\section{REFERENCES}

[1] B. Mandal, L. Li, G. S. Wang and J. Lin. Towards Detection of Bus Driver Fatigue Based on Robust Visual Analysis of Eye State, in IEEE Transactions on Intelligent Transportation Systems, vol. 18, no. 3, pp. 545-557, March 2017.

[2] National Informatics center report on road accidents: https://morth.nic.in/sites/default/files/Road_Accidednts .pdf

[3] P. Ingalepatil, P. Barhate, B. Nemade and V. D. Chaudhari, Alcohol DetectionSystemin Vehicle Using Arduino, International Research Journal of Engineering and Technology(IRJET), vol. 04, no. 06, 2017.

[4] Nawaz D. Artificial Intelligence Face Recognition for applicant tracking system. International Journal of Emerging Trends in Engineering Research. 2020; $7(12)$.

doi:https://doi.org/10.30534/ijeter/2019/277122019

[5] Soukupov'a T, Cech J. Real-Time Eye Blink Detection Using Facial Landmarks. Luka Cehovin, Rok Mandeljc, Vitomir`Struc (eds.) Rimske Toplice, Slovenia,; 2016.

[6] N. SHINDE, A. GIRI, S. RIMA and P. SINGH, ALCOHOL DETECTION AND VEHICLE ENGINE LOCKING SYSTEM, International Journal of Industrial Electronics and Electrical Engineering, vol. 6, no. 3, 2018.

[7] Africa A. Global Positioning System Using Mobile Phones: Three-Dimensional Positioning. International Journal of Emerging Trends in Engineering Research. 2020;8(2):295-299. doi:10.30534/ijeter/2020/09822020

[8] A. Rosebrock, Drowsiness detection with OpenCV PyImageSearch, PyImageSearch, 2017

[9] D. A. Preetham, M. S. Rohit, A. G. Ghontale and M. J. P. Priyadarsini, Safety helmet with alcohol detection and theft control for bikers, 2017 International Conference on Intelligent Sustainable Systems (ICISS), Palladam, 2017, pp. 668-673.

[10] B. Warwick, N. Symons, X. Chen and K. Xiong, Detecting Driver Drowsiness Using Wireless Wearables, 2015 IEEE 12th International Conference on Mobile Ad Hoc and Sensor Systems, Dallas, TX, 2015, pp. 585-588.

[11] G. Rajesh, Alcohol Detection and Alert System in Vehicles using Arduino, International Journal for Research in Applied Science and Engineering Technology, vol. 7, no. 4, 2019, pp. 1166-1169. 